\title{
Regioselective Oxidative Cleavage of Benzylidene Acetals of Glycopyranosides with Periodic Acid Catalyzed by Tetrabutylammonium Bromide
}

\author{
Jean-Michel Vatèle* \\ Université Lyon 1, Institut de Chimie et Biochimie Moléculaires et Supramoléculaires (ICBMS), UMR 5246 CNRS, Equipe SURCOOF, \\ bât. Raulin, 43, Bd. du 11 Novembre 1918, 69622 Villeurbanne Cedex, France \\ E-mail: vatele@univ-lyon1.fr \\ Received: 03.09.2013; Accepted after revision: 01.10.2013 \\ Dedicated to the memory of Professor André Lubineau
}

\begin{abstract}
A combination of periodic acid, tetrabutylammonium bromide, and wet alumina in dichloromethane efficiently oxidized benzylidene acetals of carbohydrates to the corresponding hydroxybenzoates in excellent yields $(>90 \%)$. Under these conditions, other protecting groups, such as tert-butyl(dimethyl)silyl, tert-butyl(diphenyl)silyl, and functional groups, such as epoxide, were unaffected. By varying the nature of the protecting group at the $\mathrm{C} 3$ position, good to high regioselectivity toward 4- or 6-benzoates was obtained.
\end{abstract}

Key words: acetals, carbohydrates, oxidation, cleavage, regioselectivity, protecting groups

It is well known that carbohydrates play important roles, not only as partial structures of various biologically active compounds, but also as inexpensive starting materials for total syntheses of natural products. ${ }^{1}$ Regioselective protection of individual hydroxy groups of carbohydrates is an essential step in the synthesis of oligosaccharides. ${ }^{2}$ Discrimination among the various secondary hydroxy groups of a carbohydrate is often a difficult task. The regioselective cleavage of benzylidene acetals is an appealing approach to attaining this goal. These acetals are widely used as protecting groups for 1,2- and 1,3-diols in organic synthesis because of their ease of preparation and their tolerance to a wide variety of reagents and reaction conditions. Although many methods are available for the reductive opening of benzylidene acetals to give $O$-benzyl ethers, ${ }^{3}$ this is not the case with regard to their oxidative cleavage to form hydroxybenzoates. Benzylidene acetals can be oxidatively cleaved by using trityl tetrafluoroborate, ${ }^{4}$ ozone, ${ }^{5}$ palladium(II) acetate or copper(II) chloride/tert-butyl hydroperoxide, ${ }^{6} \mathrm{~N}$-bromosuccinimide and water, ${ }^{7}$ 2,3-dichloro-5,6-dicyano-1,4-benzoquinone and water, ${ }^{8}$ bipyridinium chlorochromate and 4-chloroperoxybenzoic acid, ${ }^{9}$ sodium or potassium bromate and sodium dithionite, ${ }^{10}$ cobalt(II) acetate, $N$-hydroxysuccinimide, and dioxygen, ${ }^{11}$ or dimethyldioxirane. ${ }^{12 a, b}$ These methods are not frequently used because they require harsh conditions, long reaction times, or ecologically unfriendly reagents, and because most of them give only moderate to low regioselectivities.

SYNLETT 2014, 25, 0115-0119

Advanced online publication: 08.11.2013

DOI: 10.1055/s-0033-1340056; Art ID: ST-2013-D0847-L

(C) Georg Thieme Verlag Stuttgart · New York
Here, we describe a new procedure for high-yielding regioselective cleavage of benzylidene-protected glycosides to give the corresponding hydroxybenzoates by using a periodic acid, tetrabutylammonium bromide and wet alumina system.

In continuation in our interest in the development of novel synthetic applications of systems consisting of $(2,2,6,6-$ tetramethylpiperidin-1-yl)oxyl (TEMPO) and co-oxidants, ${ }^{13}$ and encouraged by reports on oxidative deprotection of $O$-benzyl ethers by TEMPO and sodium hypochlorite ${ }^{14}$ or its oxoammonium salts,$^{15}$ we examined the oxidative cleavage of the 4,6-O-benzylidene derivative 1 with TEMPO and various co-oxidants. However, regardless of the conditions that we used, such as TEMPO, iodosylbenzene and Lewis acid ${ }^{13 b, c, f}$ or TEMPO, copper(II) bromide and dioxygen, ${ }^{13 \mathrm{~d}}$ either no reaction occurred or partial hydrolysis of the benzylidene group was observed.

With the aim of overcoming the drawbacks of existing methods, we screened a number of oxidizing systems that might promote the transformation of compound $\mathbf{1}$ into the corresponding hydroxybenzoates $\mathbf{1 a}, \mathbf{b}$, and our most significant results are listed in Table 1.

Hypervalent iodine reagents in conjunction with bromide salts have been used for the oxidative cleavage of simple acetals. ${ }^{16}$ 1,2-Dihydroxy-1,2-dihydro-3H-1 $\lambda^{3}$-benziodol3 -one 1-oxide (IBX) or diacetyl(phenyl)- $\lambda^{3}$-iodane $\left[\mathrm{PhI}(\mathrm{OAc})_{2}\right]$ in the presence of tetrabutylammonium bromide in aqueous acetonitrile at $65{ }^{\circ} \mathrm{C}$ gave the desired 4and 6-O-benzoates in acceptable yields, but with long reaction times and modest degrees of regioselectivity (Table 1, entries 1 and 2). Marcotullio and co-workers have reported an oxidative cleavage of acetals with Oxone supported on wet alumina. ${ }^{17}$ Under their conditions, we found almost no reaction occurred, and a mixture of benzoates was obtained in low yield (entry 3). Conversely, in the presence of 0.5 equivalents of tetrabutylammonium bromide, supported Oxone gave a roughly 1:1 mixture of regioisomers in good yield (entries 4 and 5). Interestingly, the use of 1,2-dichloroethane as the solvent instead of acetonitrile reversed the regioselectivity to give the 6-Obenzoate 1a as the main product in $60 \%$ yield (entry 6). Replacing Oxone with periodic acid ${ }^{18}$ and performing the reaction in dichloromethane allowed the cleavage to proceed efficiently ( $90 \mathrm{~min}$, r.t.) to give a mixture of diastereo- 
Table 1 Optimization of Conditions for the Oxidative Cleavage of Acetal 1

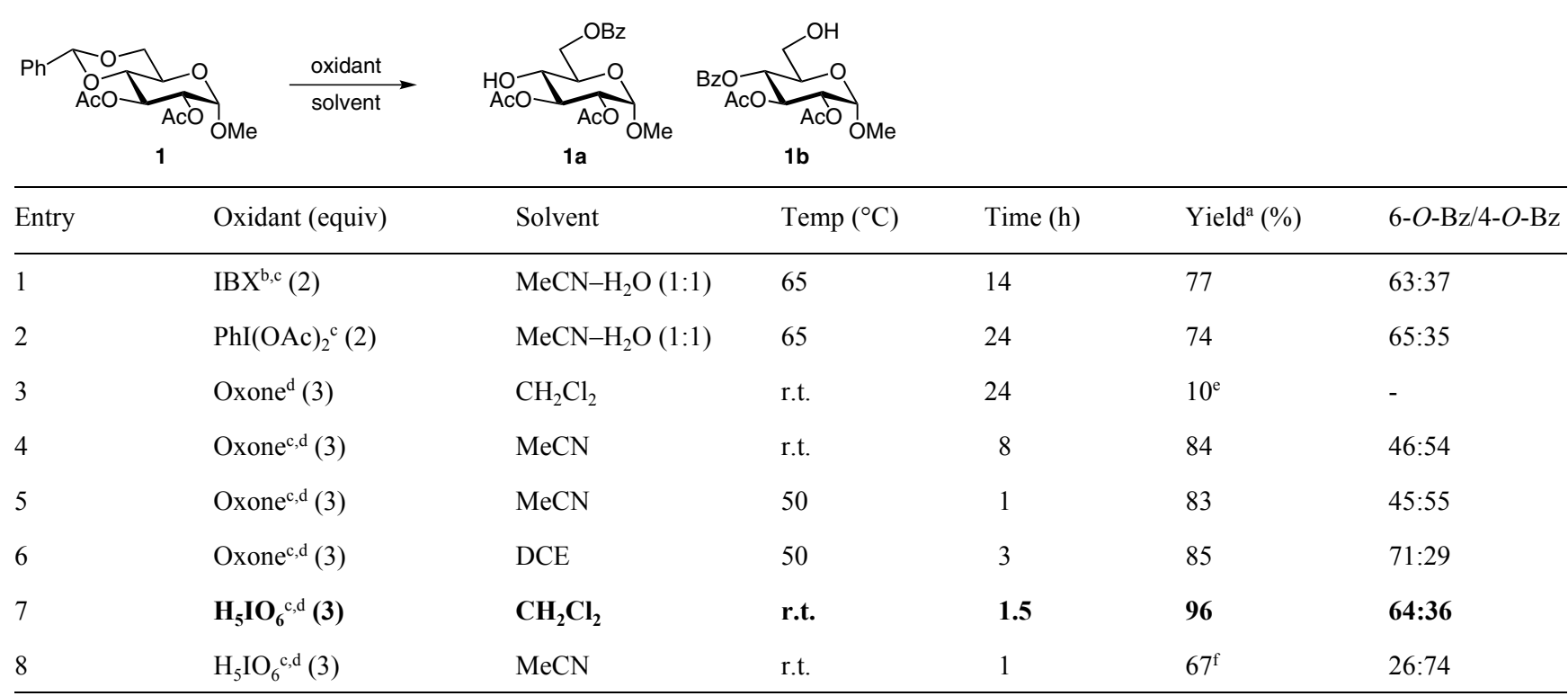

${ }^{a}$ Isolated yield.

${ }^{\mathrm{b}}$ IBX $=1$,2-dihydroxy-1,2-dihydro-3 $H$-1 $1 \lambda^{3}$-benziodol-3-one 1-oxide.

${ }^{\mathrm{c}}$ In the presence of TBAB ( 0.5 equiv).

${ }^{\mathrm{d}}$ In the presence of wet $\mathrm{Al}_{2} \mathrm{O}_{3}(2.2 \mathrm{~g} / \mathrm{mmol}$ of substrate $)$.

${ }^{\text {e }}$ Starting material was recovered (74\%).

${ }^{\mathrm{f}}$ Partial hydrolysis of the 4,6-O-benzylidene group was observed.

mers in excellent yield (96\%), but with a moderate regioselectivity (64:36) in favor of the 6-O-benzoate 1a. In acetonitrile, the reverse regioselectivity in favor of the 4$O$-benzoate $\mathbf{1 b}$ was observed, showing that, as in the case of Oxone as oxidant, the solvent has a significant effect on the regioselectivity of the cleavage of the 4,6-O-benzylidene moiety of $\mathbf{1}$ (entry 8 ).

We next examined the scope of the oxidative cleavage of various benzylidene acetals and the effects of the nature of protecting groups on the regioselectivity of the reaction of various monosaccharides with periodic acid/tetrabutylammonium bromide/wet alumina in dichloromethane. ${ }^{19,20}$ As can be seen in Table 2, the yield of the oxidative cleavage was in excess of $90 \%$ with most substrates. We first studied the effect of the nature of the protecting groups at $\mathrm{C}-2$ and $\mathrm{C}-3$ on the regioselectivity of the reaction of methyl 4,6-O-benzylidene- $\alpha$-D-glucopyranoside derivatives. Among the esters screened (entries 1-3), the dichloroacetyl group gave the best regioselectivity in favor of the 6-O-benzoate $\mathbf{3 b}$ (entry 3 ), in a similar manner to the corresponding reaction with dimethyldioxirane. ${ }^{12 a}$ Bulky electron-donating groups in the 3-position, such as tertbutyl(dimethyl)silyl, tert-butyl(diphenyl)silyl, or tosyl, induced a reversal of the selectivity, with a high preference for the formation of the secondary benzoate (entries 6,8 , and 9), once again in agreement with the corresponding reaction with dimethyldioxirane. ${ }^{12 a}$ Protected methyl 2 -deoxy-2- phthalimido- $\beta$-D-glucopyranoside derivatives gave similar regioselectivities to those obtained in the $\alpha$ glucopyranoside series, confirming the predominant role of the substituent at O-3 on the regioselectivity of the ox- idative cleavage of 4,6-benzylidene acetals (entries 1113 ). As seen in the manno and galacto series, the regioselectivity was not affected by the configuration at $\mathrm{C} 4$ or $\mathrm{C} 2$ (entries 14-16). Oxidative cleavage of the exo- and endodibenzylidene mannopyranoside 17 (entries 17 and 18) gave two products, both having a 2-O-benzoate group, indicating that cleavage of the 2,3-O-benzylidene group of $\mathbf{1 7}$ led to the formation of the axially oriented benzoate group, in agreement with the regioselectivity observed with the potassium bromate/sodium dithionite system. ${ }^{10 \mathrm{~b}}$ Cleavage of the acid-sensitive 4,6-(4-methoxybenzylidene) acetal 18 gave an equal mixture of the two regioisomers in $75 \%$ yield, along with $20 \%$ of methyl 2,3 -di- $O$ acetyl- $\alpha$-D-glucopyranoside (entry 19 ). Oxidation of compound 19, bearing an epoxy function, gave a 1:1 mixture of regioisomers in high yield, although periodic acid is known to cleave epoxides to form carbonyl derivatives (entry 20). ${ }^{21}$ In the presence of the periodic acid/bromide system, compound 20, bearing two benzyl groups, underwent concomitant oxidative cleavage of the benzyl and benzylidene groups to give several products, as observed by TLC. ${ }^{22}$ By using Oxone and tetrabutylammonium bromide in aqueous acetonitrile, the two deprotected regioisomers 20a and 20b could be obtained in $68 \%$ yield (entry 22).

To elucidate the mechanism of our oxidative cleavage of benzylidene acetals, we conducted some additional experiments on compound $\mathbf{1}$. We found that oxidation of $\mathbf{1}$ was inhibited by galvinoxyl free radical or TEMPO $(0.1$ equiv), or by performing the reaction in darkness. To trap a putative hemi-orthoester or oxonium ion, we carried out 
the reaction in the presence of a large excess of methanol and, in addition to $\mathbf{1 a}, \mathbf{b}$ ( $40 \%$ yield), we obtained the orthoester 21 in $24 \%$ yield (Scheme 1). The structure and configuration of 21 were assigned by means of NMR experiments (COSY, HSQC, and NOE). Under our reaction conditions, pure hydroxybenzoate $\mathbf{1 b}$ was not converted to any extent into its regioisomer $1 \mathbf{a}$ by equilibration. On the basis of these results, we propose a plausible mechanism for the cleavage of benzylidene acetals with periodic acid and tetrabutylammonium bromide, which is shown in Scheme 1. Oxidation of bromide ion by periodic acid gives molecular bromine, ${ }^{23}$ as evidenced by the orange color of the suspension. The bromine molecule undergoes homolytic cleavage under visible light to generate a bromine radical. Benzylic hydrogen abstraction gives radical A which is trapped by the bromine radical to give the bromo acetal intermediate B. ${ }^{24}$ Disproportionation $\mathbf{B}$ to benzoxonium ion $\mathbf{C}$ and bromide ion, followed by attack by water, gives hemi-orthoester $\mathbf{D}$, which collapses to provide the 4- and 6-O-benzoates. In the presence of methanol, the oxocarbenium ion $\mathbf{C}$ gives the isolable orthoester $\mathbf{E}$, which is hydrolyzed to form hydroxybenzoates. The role of alumina is probably in buffering the acidity of the periodic acid, thereby preventing hydrolysis of the benzylidene acetal, and in mediating the reaction by dispersion of the oxidant on its surface. ${ }^{25}$

Table 2 Oxidative Cleavage of Benzylidene Acetals of Carbohydrate Derivatives with the Periodic Acid/Tetrabutylammonium Bromide/Wet Alumina System

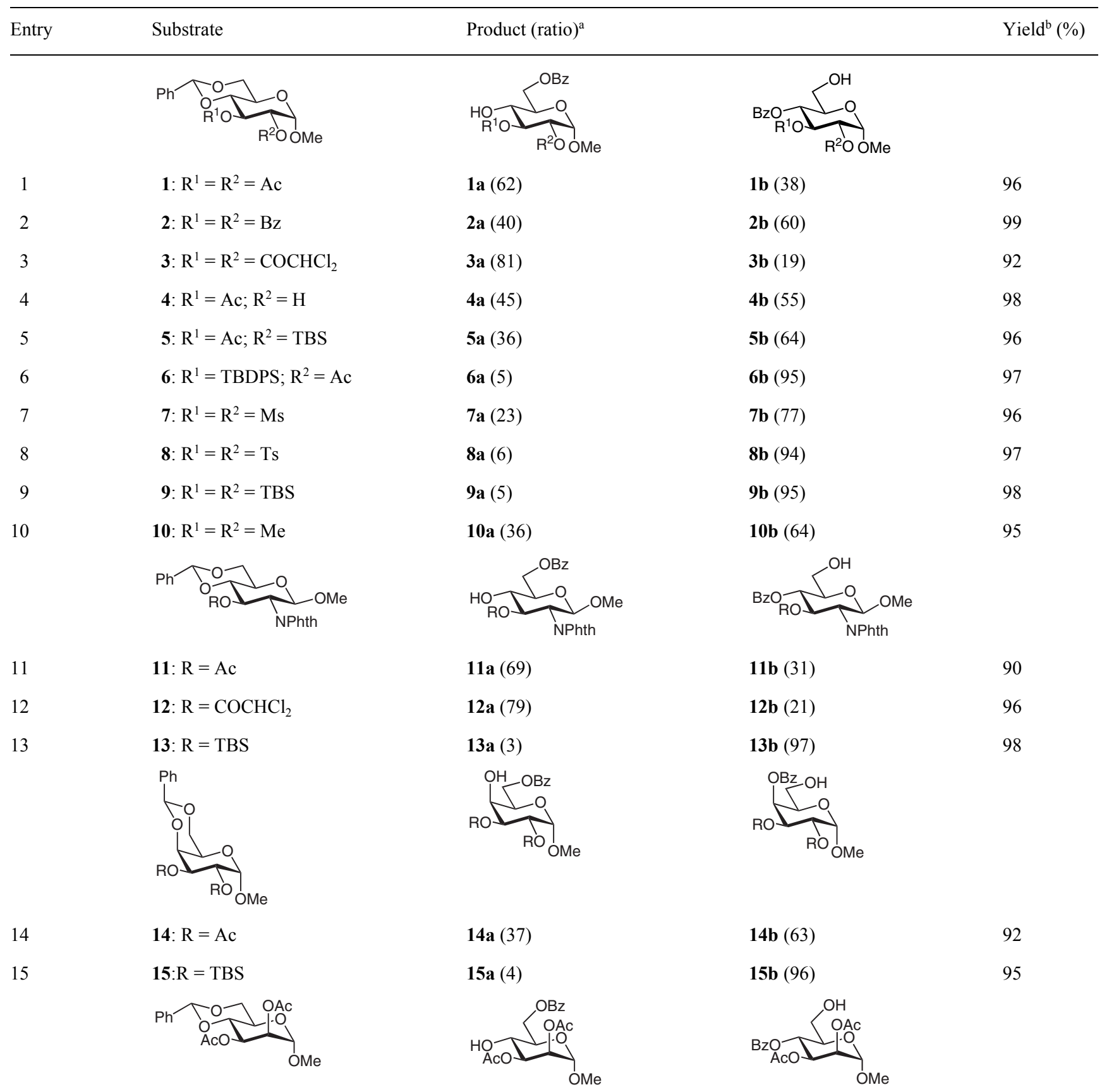


Table 2 Oxidative Cleavage of Benzylidene Acetals of Carbohydrate Derivatives with the Periodic Acid/Tetrabutylammonium Bromide/Wet Alumina System (continued)

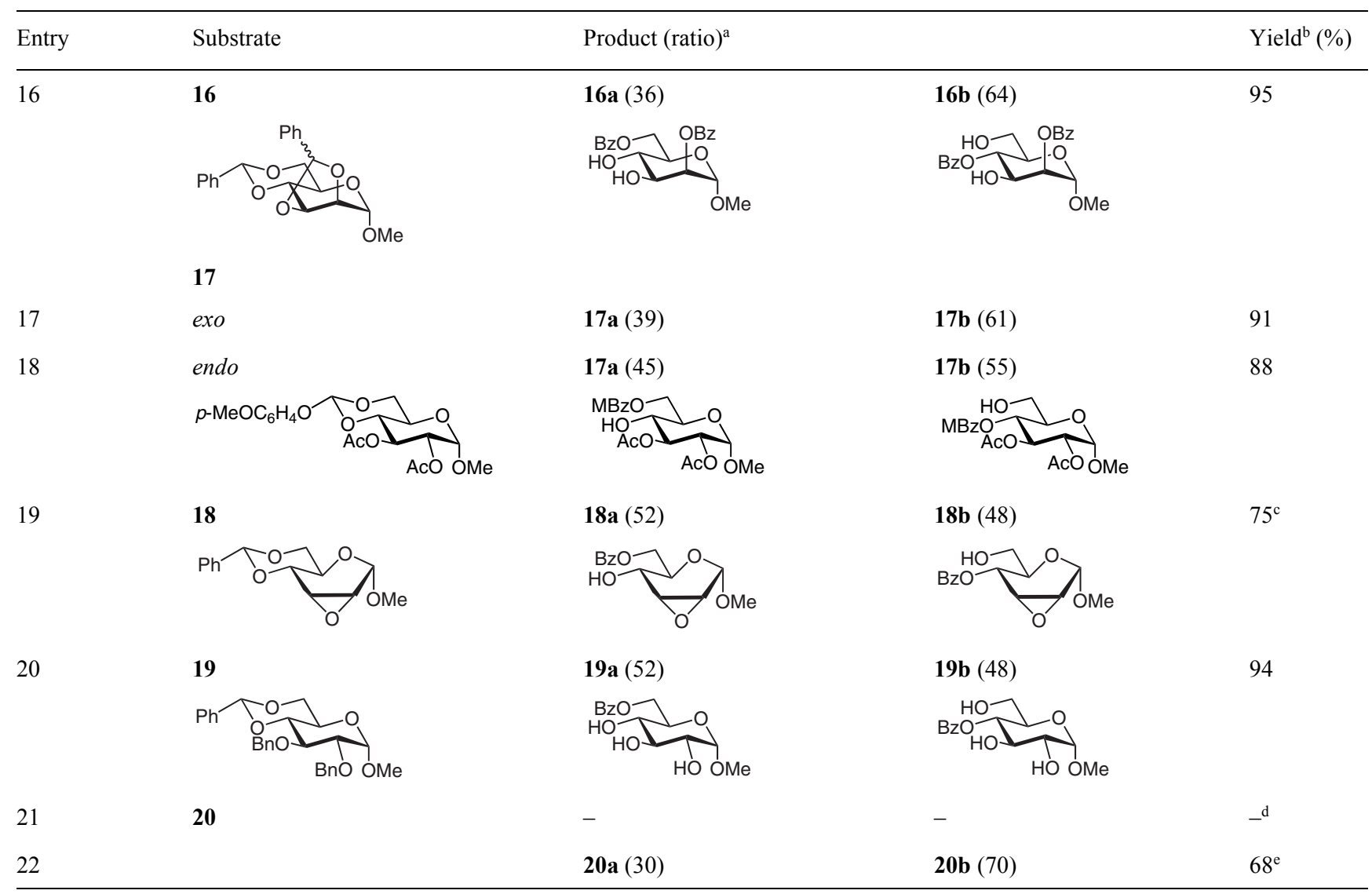

${ }^{a}$ The ratio of the two regioisomers was determined by ${ }^{1} \mathrm{H}$ NMR spectroscopy of the pure mixture.

${ }^{\mathrm{b}}$ Isolated yield.

${ }^{c}$ The product of $p$-anisylidene hydrolysis was isolated (20\%).

d An intractable mixture of products was formed.

${ }^{\mathrm{e}}$ Reaction conditions: substrate ( $\left.1 \mathrm{mmol}\right)$, Oxone ( $3 \mathrm{~g}, 5$ equiv), TBAB ( $0.32 \mathrm{~g}, 0.5$ equiv), wet alumina ( $\left.3.8 \mathrm{~g}\right), \mathrm{MeCN}(10 \mathrm{~mL})$, r.t., $10 \mathrm{~h}$.

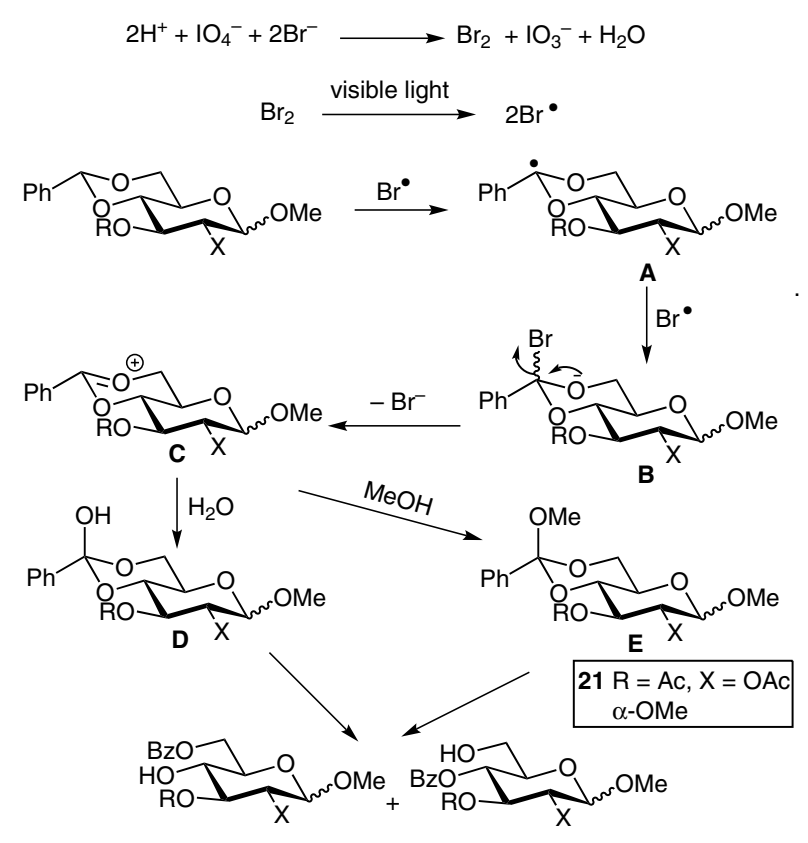

Scheme 1 Proposed mechanism for oxidative cleavage of benzylidene acetals mediated by periodic acid, tetrabutylammonium bromide, and wet alumina
In summary, we have shown that the periodic acid/tetrabutylammonium bromide/wet alumina system is an efficient reagent system for regioselective oxidative cleavage of a variety of benzylidene acetals of carbohydrates, and that the reaction is controlled by the nature of the protecting group at the $\mathrm{C} 3$ position. Because the reaction involves mild conditions, requires cheap reagents, is chemoselective, has short reaction times, and involves simple workup, this protocol represents a valuable alternative to existing methods. Studies of other uses of this new supported oxidizing system are currently under way.

\section{Acknowledgment}

We thank Dr. Dominique Lafont for a gift of 1,3,4,6-tetra- $O$-acetyl2-deoxy-2-phthalimido- $\beta$-D-glucopyranose.

Supporting Information for this article is available online at http://www.thieme-connect.com/ejournals/toc/synlett. 


\section{References and Notes}

(1) Hanessian, S. Total Synthesis of Natural Products: The 'Chiron' Approach; Pergamon: Oxford, 1983.

(2) (a) Greene, T. W.; Wuts, P. G. M. Protective Groups in Organic Synthesis; Wiley-Interscience: New York, 1999, 3rd ed.. (b) Protecting Groups; Kocieńsky, P. J., Ed.; Thieme: Stuttgart, 2005, 3rd ed.

(3) (a) Preparative Carbohydrate Chemistry; Hanessian, S., Ed.; Marcel Dekker: New York, 1997. (b) Ohlin, M.; Johnsson, R.; Ellervik, U. Carbohydr. Res. 2011, 346, 1358; and references cited therein.

(4) Hanessian, S.; Staub, A. P. A. Tetrahedron Lett. 1973, 14, 3551.

(5) Deslongchamp, P.; Moreau, C.; Fréhel, D.; Chênevert, R. Can. J. Chem. 1975, 73, 1204.

(6) Sato, K.; Igarashi, T.; Yanagisawa, Y.; Kawauchi, N.; Hashimoto, H.; Yoshimaura, J. Chem. Lett. 1988, 1699.

(7) Binkley, R. W.; Goewey, G. S.; Johnson, J. C. J. Org. Chem. 1984, 49, 992.

(8) (a) Oikawa, Y.; Yoshioka, T.; Yonemitsu, O. Tetrahedron Lett. 1982, 23, 889. (b) Zhang, Z. Y.; Magnusson, G. J. Org. Chem. 1996, 61, 2394.

(9) Luzzio, F. A.; Bobb, R. A. Tetrahedron Lett. 1997, 38, 1733.

(10) (a) Adinolfi, M.; Barone, G.; Guariniello, L.; Iadanisi, A. Tetrahedron Lett. 1999, 40, 8439. (b) Senthilkumar, P. P.; Aravind, A.; Basharan, S. Tetrahedron Lett. 2007, 48, 1175.

(11) Chen, Y.; Wang, P. G. Tetrahedron Lett. 2001, 42, 4955.

(12) (a) Stévenin, A.; Boyer, F.-D.; Beau, J.-M. J. Org. Chem. 2010, 75, 1783. (b) Mycock, D. K.; Sherlock, A. E.; Glossop, P. A.; Hayes, C. J. Tetrahedron Lett. 2008, 49, 6390.

(13) (a) Vatèle, J.-M. Tetrahedron Lett. 2006, 47, 715. (b) Vatèle, J.-M. Synlett 2006, 2055. (c) Vatèle, J.-M. Synlett 2008, 1785. (d) Vatèle, J.-M. Synlett 2009, 2143. (e) Vatèle, J.-M. Tetrahedron 2010, 66, 904. (f) Barnych, B.; Vatèle, J.-M. Synlett 2011, 2048.

(14) (a) Cho, N. S.; Park, C. H. Bull. Korean Chem. Soc. 1994, 15, 924. (b) Cho, N. S.; Park, C. H. J. Korean Chem. Soc. 1995, 39, 657.

(15) (a) Miyazawa, T.; Endo, T. Tetrahedron Lett. 1986, 27, 3395. (b) Pradhan, P. P.; Bobitt, J. M.; Bailey, W. F. J. Org. Chem. 2009, 74, 9524.
(16) (a) Kuhakarn, C.; Panchan, W.; Chiampanichayakul, S.; Samakkanad, N.; Pohmakotr, M.; Reutrakul, V.; Jaipetch, T. Synthesis 2009, 929. (b) Panchan, W.; Chiampanichayakul, S.; Snyder, D. L.; Yodbuntung, S.; Pohmakotr, M.; Reutrakul, V.; Jaipetch, T.; Kuhakarn, C. Tetrahedron 2010, 66, 2732 .

(17) Curini, M.; Epifano, F.; Marcotullio, M. C.; Rosati, O. Synlett 1999, 777.

(18) For uses of periodic acid in conjunction with bromide salts in organic synthesis, see: Yousefi-Seyf, J.; Tajeian, K.; Kolvari, E.; Koukabi, N.; Khazaei, A.; Zolfigol, M. A. Bull. Korean Chem. Soc. 2012, 33, 2619; and references cited therein.

(19) Appropriate physical and analytical data were obtained for all new compounds (see Supporting Information).

(20) Oxidative Cleavage of Benzylidene Acetals; General Procedure

Wet alumina was prepared by mixing neutral alumina $(50 \mathrm{~g}$, Fluka ref. 06300; Brockmann activity 1) with $\mathrm{H}_{2} \mathrm{O}(10 \mathrm{~g})$ and shaking until a free-flowing homogeneous powder was obtained. The wet $\mathrm{Al}_{2} \mathrm{O}_{3}(2.2 \mathrm{~g})$, TBAB ( $0.16 \mathrm{~g}, 0.5$ equiv), and $\mathrm{H}_{5} \mathrm{IO}_{6}(0.68 \mathrm{~g}, 3$ equiv) were added successively to a solution of the benzylidene acetal $(1 \mathrm{mmol})$ in $\mathrm{CH}_{2} \mathrm{Cl}_{2}(10$ $\mathrm{mL}$ ), and the suspension was vigorously stirred at r.t. for 90 min. The resulting orange suspension was poured onto a column of silica gel and the hydroxybenzoates were eluted with an appropriate mixture of PE and EtOAc.

(21) Mori, K. Tetrahedron 1977, 33, 289.

(22) Under the same reaction conditions $\left[\mathrm{H}_{5} \mathrm{IO}_{6}\right.$ (3 equiv), TBAB ( 0.5 equiv), wet alumina, r.t., $90 \mathrm{~min}]$, 3-O-benzyl-1,2:5,6di-O-isopropylidene- $\alpha$-D-glucofuranose gave the debenzylated product in $81 \%$ yield.

(23) Koukabi and co-workers have demonstrated by UV studies that $\mathrm{Br}_{2}$ is formed in a mixture of $\mathrm{H}_{5} \mathrm{IO}_{6}$ and $\mathrm{NaBr}$ in water; see ref. 18 .

(24) A bromoacetal intermediate has been proposed for the oxidative cleavage of benzylidene acetals with $\mathrm{NBS}$ in $\mathrm{CCl}_{4}$; see: Hanessian, S.; Plessas, N. R. J. Org. Chem. 1969, 34, 1035.

(25) For an example of a study on the mechanism of $\mathrm{Al}_{2} \mathrm{O}_{3}$ mediated oxidation, see: Kropp, P. J.; Breton, G. W.; Fields, J. D.; Tung, J. C.; Loomis, B. R. J. Am. Chem. Soc. 2000, 122,4280 . 